\title{
Murine erythroid short-term radioprotection requires a BMP4-dependent, self-renewing population of stress erythroid progenitors
}

\author{
Omid F. Harandi,1,2 Shailaja Hedge,1,3 Dai-Chen Wu,4 Daniel Mckeone,5 and Robert F. Paulson'1,2,3,5 \\ ${ }^{1}$ Center for Molecular Immunology and Infectious Disease, ${ }^{2}$ Graduate Program in Genetics at the Huck Institutes for the Life Sciences, \\ ${ }^{3}$ Department of Veterinary and Biomedical Sciences, ${ }^{4}$ Graduate Program in Biochemistry, Microbiology, and Molecular Biology, \\ and ${ }^{5}$ Schreyer Honors College, Pennsylvania State University, University Park, Pennsylvania, USA.
}

\begin{abstract}
Acute anemic stress induces a systemic response designed to increase oxygen delivery to hypoxic tissues. Increased erythropoiesis is a key component of this response. Recovery from acute anemia relies on stress erythropoiesis, which is distinct from steady-state erythropoiesis. In this study we found that the bone morphogenetic protein 4-dependent (BMP4-dependent) stress erythropoiesis pathway was required and specific for erythroid short-term radioprotection following bone marrow transplantation. BMP4 signaling promoted the development of three populations of stress erythroid progenitors, which expanded in the spleen subsequent to bone marrow transplantation in mice. These progenitors did not correspond to previously identified bone marrow steady-state progenitors. The most immature population of stress progenitors was capable of self renewal while maintaining erythropoiesis without contribution to other lineages when serially transplanted into irradiated secondary and tertiary recipients. These data suggest that during the immediate posttransplant period, the microenvironment of the spleen is altered, which allows donor bone marrow cells to adopt a stress erythropoietic fate and promotes the rapid expansion and differentiation of stress erythroid progenitors. Our results also suggest that stress erythropoiesis may be manipulated through targeting the BMP4 signaling pathway to improve survival after injury.
\end{abstract}

\section{Introduction}

Steady-state erythropoiesis occurs in the bone marrow and is primarily homeostatic. New erythrocytes are continuously generated at a constant rate. The situation is dramatically different during embryogenesis and in response to acute anemic stress in adults. During these times, stress erythropoiesis predominates, which rapidly generates new erythrocytes at a rate beyond the capability of steady-state erythropoiesis $(1,2)$. The mechanisms that regulate this response are distinct from those that regulate steadystate erythropoiesis. In response to anemic stress, the microenvironments of the fetal liver and adult spleen and liver promote the expansion of a specialized population of stress erythroid progenitors (3-7). Previous work has identified a mechanistic link between fetal liver erythropoiesis and adult stress erythropoiesis through the analysis of the murine flexed-tail $(f)$ mutation (8-11). $f / f$ mutant mice exhibit near-normal steady-state erythropoiesis but are defective in stress erythropoiesis. Our analysis of these mice showed that they have a defect in the expansion of a specialized population of stress erythroid progenitors in the spleen, which we term "stress BFU-Es" (3). These progenitors have a greater ability to generate large numbers of new erythrocytes faster than bone marrow steady-state progenitors (5). We identified a mutation in Smad5 in $f$ mutant mice $(3,12)$, which implicated bone morphogenetic protein (BMP) signaling in stress erythropoiesis. We showed that BMP4 drives the expansion of stress BFU-Es in the spleen during the recovery from acute anemia. The $f$ mutation causes a defect in Smad5 splicing, which leads to the tissue-specific expression of a dominant-negative form of Smad5. This mutated version of Smad5 inhibits BMP4 signaling by Smad1, -5 , and $-8(3,12)$.

Conflict of interest: The authors have declared that no conflict of interest exists. Citation for this article: J Clin Invest. 2010;120(12):4507-4519. doi:10.1172/JCI41291.
In addition to BMP4, the expansion of stress BFU-Es relies on the action of 2 additional signals, SCF and hypoxia. These 3 signals act in concert and are necessary and sufficient to recapitulate in vitro the 45-fold expansion of stress BFU-Es observed in the spleen during the recovery from acute anemia in vivo (5). In vitro culture of spleen cells showed that the action of these 3 factors results in the expansion of a distinct stress erythroid progenitor cell $\left(\mathrm{Kit}^{+} \mathrm{CD} 71^{+} \mathrm{Ter} 119^{+}\right)$, which expresses immature progenitor (Kit) and erythroid lineage-specific markers (Ter119). Previously we demonstrated that the stress erythroid progenitors in the spleen were completely mobilized in response to acute anemia. Following initial recovery it takes an additional 2 weeks to replenish the stress progenitor population. Our analysis showed that bone marrow cells repopulate the spleen, and hedgehog- and BMP4-dependent signals are required for the development of new erythroid stress progenitors (13). Based on these observations, we developed a model in which acute anemia leads to tissue hypoxia, which induces BMP4 expression in the spleen. These 2 signals plus SCF drive the rapid expansion of stress progenitors, which differentiate into new erythrocytes. Following recovery, bone marrow cells migrate to the spleen and develop into new stress erythroid progenitors primed to respond to the next anemic challenge.

Our previous work utilized phenylhydrazine-induced (PHZinduced) acute hemolytic anemia as a model system to study stress erythropoiesis. This model, however, has several limitations. First, PHZ treatment only affects the erythroid lineage, so it was not possible to analyze the potential of stress erythroid progenitors to develop into other myeloid lineages. Second, the analysis of stress erythropoiesis in the PHZ model is focused on late-stage events, the expansion and differentiation of stress progenitors resident in the spleen. For this report, we utilized a robust experimental system that allows us to analyze all steps in the BMP4-dependent stress 
erythropoiesis pathway and study the development of stress erythroid progenitors in vitro and in vivo. This system takes advantage of the observation that new erythrocytes must be rapidly generated immediately following bone marrow transplant. Although a single stem cell is sufficient to reconstitute the hematopoietic system of an irradiated mouse, in practice, short-term radioprotective cells must also be transplanted along with stem cells to alleviate the lethal anemia and potential for infection or hemorrhage caused by the myeloablative conditioning prior to transplant $(14,15)$. In this study, we demonstrate that defects in the BMP4-dependent stress erythropoiesis pathway lead to a specific delay in erythroid shortterm radioprotection, while the generation of platelets and neutrophils was unaffected. We show that spleen stress erythroid progenitors are derived from short-term reconstituting HSCs (STR-HSCs), which are characterized as $\mathrm{CD} 34^{+} \mathrm{Kit}^{+} \mathrm{Sca} 1^{+} \mathrm{Lin}^{-}$cells. Once in the spleen, they develop into 3 distinct stress erythroid progenitor populations, each with a different erythroid potential. Donor cells with defects in BMP4 signaling $(f / f)$ when transplanted at low dose $\left(10^{5}\right)$ fail to provide erythroid short-term radioprotection, which results in a lethal anemia. At higher doses $\left(5 \times 10^{5}\right)$, mutant donor cells exhibit a delayed erythroid recovery, which was associated with the delayed expression of Scl and Gata2. Overexpression of either of these molecules in mutant donor cells rescued this defect. We further characterized the spleen stress erythroid progenitors and showed that the most immature population of these progenitors, when transplanted into irradiated secondary and tertiary recipients, is capable of maintaining erythropoiesis with no contribution to other myeloid or lymphoid lineages until surviving recipient stem cells recovered. Despite the fact that recipient HSCs have repopulated these mice, donor stress erythroid progenitors rapidly responded to $\mathrm{PHZ}$-induced acute anemia. These data demonstrate that the BMP4-dependent stress erythropoiesis pathway generates a population of self-renewing stress erythroid progenitor cells that can maintain erythropoiesis in the absence of HSCs and represent an anemic stress response compartment.

\section{Results}

BMP4-dependent signaling is required and specific for erythroid short-term radioprotection following bone marrow transplant. In the immediate posttransplant recovery period, short-term radioprotective cells must rapidly produce new erythrocytes, platelets, and neutrophils. We tested whether the BMP4-dependent stress erythropoiesis pathway regulated the development of new erythrocytes following transplant and whether BMP4-dependent signaling regulated the development of other myeloid lineages, in particular megakaryocytes and neutrophils, during the recovery from transplant. We transplanted $10^{5}$ bone marrow cells isolated from C57BL/6 or C57BL $/ 6-f / f$ mice, which carry the CD45.2 allele, into lethally irradiated B6.SJL-Ptpr$c^{a} P e p 3^{b} /$ BoyJ recipients $\left(\mathrm{CD} 45.1^{+}\right)$and followed their recovery over a 26 -day period. Ninety percent $(9 / 10)$ of mice transplanted with control bone marrow survived, but none $(0 / 10)$ of the mice transplanted with mutant bone marrow lived longer than 20 days (Figure 1A). Analysis of hematocrit during the recovery period showed that control transplanted mice reached a nadir at 14 days following transplant, after which their hematocrits steadily increased. The mutant transplants failed to increase their hematocrits at any time following transplant (Figure 1B). This defect was further demonstrated when we examined reticulocyte production, which is a direct measure of new erythropoiesis. Control transplanted mice exhibited a robust reticulocyte response that paralleled their hematocrit recov- ery, while $f / f$ transplanted mice showed no reticulocyte development (Figure 1C). These data demonstrate that, at limiting numbers of transplanted cells, $f / f$ mutant donor bone marrow cells are unable to provide erythroid short-term radioprotection.

In order to overcome the lethality, we increased the number of donor bone marrow cells to $5 \times 10^{5}$. At this dose, we observed that all transplanted mice survived regardless of whether they received control or mutant bone marrow cells. Despite their survival, mice transplanted with $f / f$ mutant bone marrow exhibited a delayed erythroid recovery (Figure 1D). When we examined different erythroid parameters, the $f / f$ transplanted mice exhibited a significant delay in all parameters, which was characterized by significantly lower nadir values and delayed recovery (Supplemental Figure 1, A-C; supplemental material available online with this article; doi:10.1172/JCI41291DS1). In addition, the onset of the expansion of donor cells in the spleen was delayed and the overall expansion of cells in the spleen was diminished in mice transplanted with $f / f$ bone marrow (Supplemental Figure 1, D and E).

In contrast to the defects in erythroid recovery, mice transplanted with $f / f$ donor cells generated new platelets, neutrophils, lymphocytes, and white blood cells equally as well as control transplanted mice (Supplemental Figure 1, F-I). Flow cytometry and histological analysis showed that the number of donor-derived megakaryocytes $\left(\mathrm{CD} 45.2^{+} \mathrm{CD} 61^{+}\right)$in spleens of $f / f$ and control transplanted mice were not significantly different (Supplemental Figure 1, J and K).

The delayed recovery observed in $f / f$ transplants was not due to a generalized defect in HSCs. $f / f$ bone marrow cells are as capable as control bone marrow cells in reconstituting hematopoiesis long term. Four months after transplant, $f / f$ and control transplanted mice exhibited similar levels of reconstitution and hematological recovery (Supplemental Figure 2). Our data are completely consistent with recent work showing that Smad1 and -5 are dispensable for long-term reconstitution $(16,17)$. Furthermore, previous analysis of several multi-potential progenitors that have been implicated in short-term radioprotection $(18,19)$ showed that these progenitors were not decreased in $f / f$ mutant mice (20).

BMP4 is expressed in the spleen during the recovery from bone marrow transplant. We next examined the expression of BMP4 in the spleen during the recovery of mice transplanted with control or $f / f$ mutant bone marrow (Supplemental Figure 3). BMP4 expression was confined to the red pulp (Supplemental Figure 3D). In control transplants, early BMP4 expression (24 hours) was associated with donor cells, but by 48 hours, recipient stromal cells in the spleen expressed BMP4. The level of BMP4 increased up to day 12, when the highest levels of BMP4 were observed. After that time the expression decreased (Supplemental Figure 3, A and B). In addition to the early expression by donor cells, we also observed donor cell expression at day 12, when the majority of BMP4 expression was donor derived. These observations are consistent with our model, showing that when bone marrow cells migrate into the spleen, they encounter a hedgehog signal, which induces them to express BMP4. Our data support a mechanism in which, on day 1 , the initial wave of progenitors migrate into the spleen, encounter hedgehog, and express BMP4, which leads to the development of BMP4-responsive stress erythroid progenitors. On the other hand, donor-derived BMP4 expression on day 12 represented a second wave of progenitors migrating to the spleen, which led to replenishment of stress progenitors after recovery (Supplemental Figure 3C).

In contrast to control transplants, $f / f$ mutant transplants exhibited a delayed and diminished expression of BMP4, which is consis- 

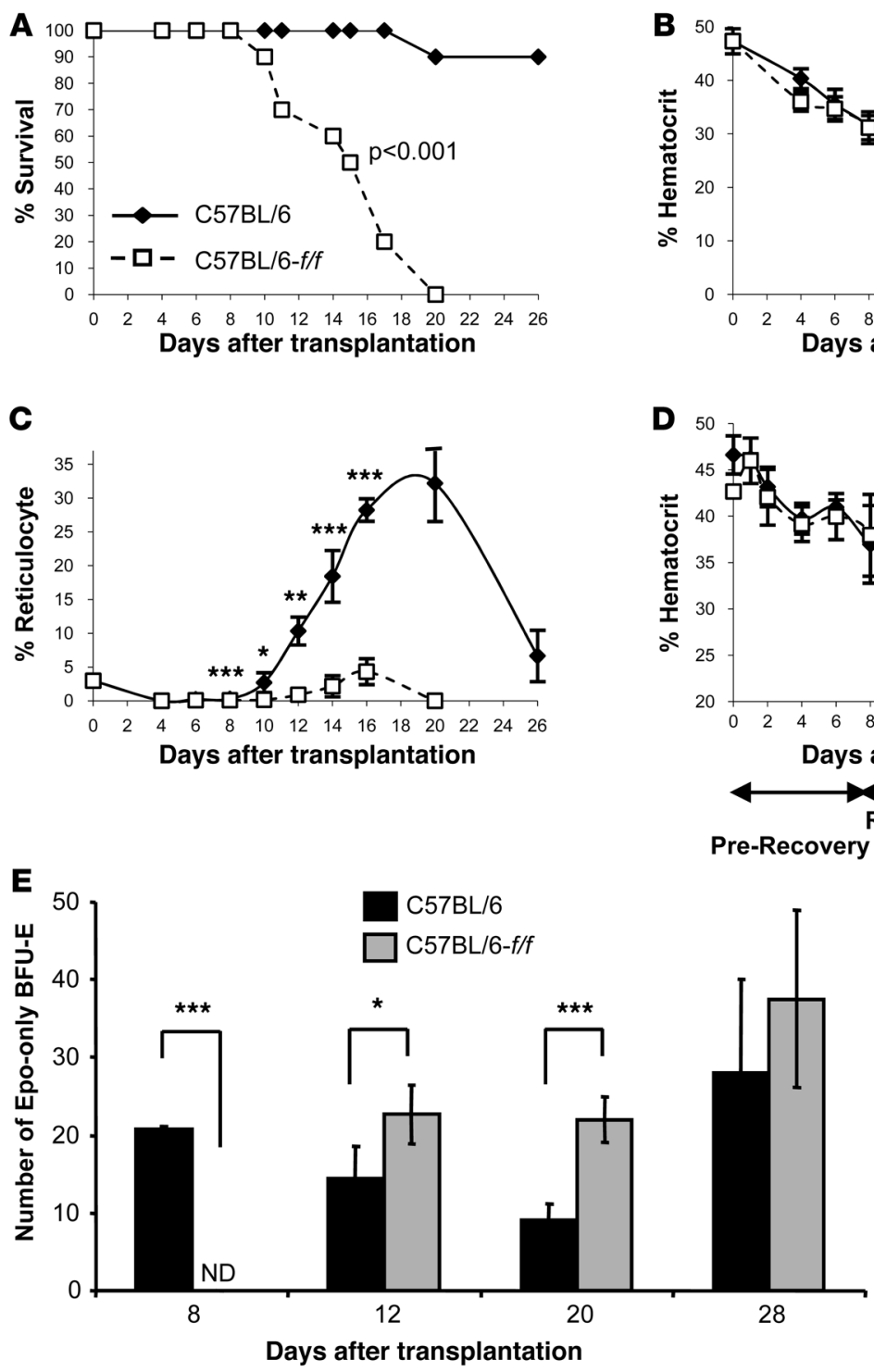
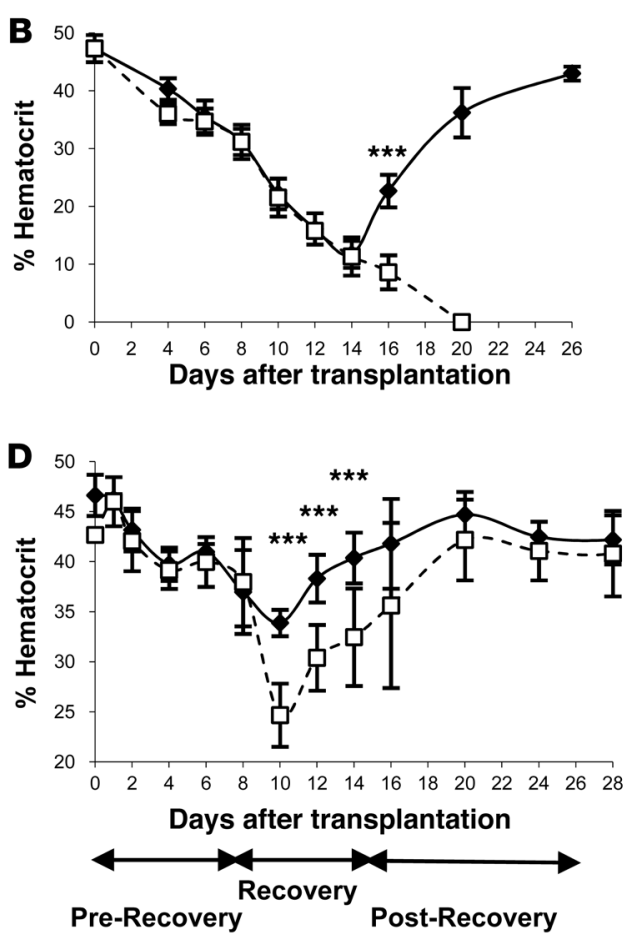

Figure 1

Mice transplanted with $f$ mutant bone marrow exhibit a defect in erythroid shortterm radioprotection. C57BL/6 mice were irradiated and transplanted with $\mathrm{C} 57 \mathrm{BL} / 6$ $\mathrm{f} / f$ mutant or $\mathrm{C} 57 \mathrm{BL} / 6$ control bone marrow. (A-C) Analysis of mice transplanted with $1 \times 10^{5}$ unfractionated bone marrow cells. (A-C) Survival of mice transplanted with mutant or control bone marrow $(\mathbf{A})$, hematocrit recovery (B), and reticulocyte production (C) following transplant. (D) Analysis of hematocrit recovery in mice transplanted with $5 \times 10^{5}$ unfractionated bone marrow cells. For each figure, each time point represents $4-18$ recipients from at least 3 independent experiments. (E) Donor-derived $\left(\right.$ CD 45.2 $\left.2^{+}\right)$spleen cells were isolated by FACS on the indicated days from CD45.1 mice that had been transplanted with $5 \times 10^{5}$ mutant or control bone marrow cells. Cells $\left(2 \times 10^{6}\right)$ were plated in methylcellulose media containing Epo only. Stress BFU-Es were scored 5 days later. No colonies were observed prior to day 8 following transplant. ND, not detected. ${ }^{*} P<0.05$; ${ }^{\star \star} P<0.01 ;{ }^{* \star *} P<0.005$. tent with our previous work on the recovery from acute anemia $(3,7)$. The expression of BMP4 by donor-derived cells was prolonged in mutant transplants. This observation suggests that defects in Smad5-dependent signaling leads to aberrant regulation of BMP4 expression by mutant donor-derived cells, which would lead to prolonged expression of BMP4 (Supplemental Figure 3).

Stress BFU-Es proliferate in the spleen during the recovery phase, but the expansion of these progenitors is delayed in mice transplanted with $\mathrm{f} / \mathrm{f}$ mutant bone marrow. One of the hallmarks of the BMP4-dependent stress erythropoiesis pathway is the expansion of stress BFU-Es in the spleen $(3,5,6)$. We examined donor-derived stress BFU-Es in the bone marrow and spleen by sorting CD $45.2^{+}$cells from the bone marrow and spleen and plating them in methylcellulose media containing only Epo, which specifically assays stress BFU-Es $(3,5)$. At no time did we observe stress BFU-Es in the bone marrow (data not shown). Until 8 days following transplant, no donor-derived stress BFU-Es were observed in the spleen (Figure 1E). Stress BFU-Es were first observed in control transplants on day 8 following transplant, but we did not detect stress BFU-Es in the spleens of mice transplanted with mutant bone marrow at this time point. However, stress BFU-Es were observed in the spleen on day 12 in mice transplanted with $f / f$ mutant bone marrow. Although the frequency of stress BFU-Es was slightly greater in $f / f$ transplanted mice, the spleens of $f / f$ transplanted mice were still significantly smaller than control transplants at this time (Supplemental Figure 1, D and E), which suggests that control transplanted mice had greater total numbers of stress BFU-Es. In contrast at day 20, the frequency of stress BFU-Es in the spleens of $f / f$ transplanted mice was greater and the spleens of $f / f$ were slightly larger than control transplanted mice (Supplemental Figure 1, D and E), which suggests that $f / f$ transplants exhibit delayed expansion of stress BFU-Es in the spleen. Later, in the post-recovery stage, at day $28, f / f$ mice had similar numbers of stress BFU-Es when compared with control mice (Figure 1E).

Overall, careful examination of data concerning the control transplants shows that the erythroid recovery in the immediate post-transplant period proceeds through 3 distinct phases. An initial stage, which we will refer to as "pre-recovery" (Days 0-8), is characterized by steadily decreasing rbc numbers and absence of 
compensatory stress erythropoiesis, as no reticulocytes are observed in the peripheral blood and spleen size is unchanged. Erythrocyte counts reach their nadir and active stress erythropoiesis occurs in a second recovery stage (Days 8-16), leading to an expansion of stress BFU-Es and an increase in rbc numbers, hemoglobin, and reticulocytes in the peripheral blood as well as an increase in spleen weight. Finally, the third stage, or post-recovery (Days 16-28), is characterized by normal hematocrit and $\mathrm{rbc}$ numbers and decreasing reticulocytes and spleen weight (shown in Figure 1D).

Three distinct populations of stress erythroid progenitors expand in the spleen during the recovery from bone marrow transplant. Previous analysis of bone marrow erythroid progenitors identified several distinct populations based on their expression of CD71 and Ter119. These cells represent late-stage erythroid progenitors that do not express Kit and do not form BFU-E colonies $(21,22)$. When we analyzed these populations in the bone marrow of mutant and control transplanted mice, we did not observe any differences in the frequencies of different progenitor populations on days 6 and 8 after transplant. However on day $10, f / f$ transplanted mice showed a decreased frequency of the most mature Ter $119^{+} \mathrm{CD} 71^{-}$cells (data not shown). Our previous work showed that BMP4, SCF, and hypoxia promote the expansion of a $\mathrm{Kit}^{+} \mathrm{CD} 71^{+} \mathrm{Ter} 119^{+}$population of spleen stress erythroid progenitors (5). Careful flow cytometry analysis of spleen cells following bone marrow transplant showed that the $\mathrm{Kit}^{+} \mathrm{CD} 71^{+} \mathrm{Ter} 119^{+}$cells could be subdivided into 3 populations with distinct staining characteristics, morphology, and erythroid potential. We term these "Populations I, II, and III". Population I cells were $\mathrm{Kit}^{+} \mathrm{CD} 71^{\mathrm{lo} / \mathrm{med}} \mathrm{Ter} 119^{\mathrm{lo} /-}$, Population II cells were $\mathrm{Kit}^{+} \mathrm{CD} 71^{\text {hiTer119med }}$, and Population III cells were $\mathrm{Kit}^{+} \mathrm{CD} 71^{\mathrm{lo} / \text { med Ter119hi }}$ (Figure 2A). We calculated the total number of each of these 3 populations during the recovery time. Population I was the primary population present at the pre-recovery stage (days 2-6), with a slight increase in the total number throughout the post-recovery time (day 16) (Figure 2, A and B). Population I was also present in non-anemic spleen (day 0), which suggests it represents the population of stress erythroid progenitors resident in the spleen (data not shown). Population I cells exhibited morphology similar to primitive progenitor cells (Supplemental Figure 4). In contrast, Population II exhibited a wave of proliferation that started at day 6 (early recovery time). We observed a 30-fold increase in these cells by day 12 (recovery time), which diminished by day 16 (post-recovery) (Figure 2, A and B). The expansion of these cells corresponded to a critical time during the recovery, which tightly correlated with the increase of spleen size at day 12 (Supplemental Figure 1D) and high production of reticulocytes (Supplemental Figure 1A). Morphological analysis of Population II showed that the cells resemble early basophilic erythroblasts (Supplemental Figure 4). Population III, with high expression of Ter119, exhibited a smaller window of appearance with significant increase in the number of cells at recovery time (day 12), which also correlated with the maximum spleen size, number of reticulocytes, and early hematocrit recovery (Supplemental Figure 1, A and D). Population III cells resembled late-stage chromatophilic and orthochromatophilic erythroblasts (Supplemental Figure 4). In addition, approximately $50 \%$ of these cells stained positive for benzidine (data not shown). Further analysis of these populations showed that Population I also contained a population of Sca $1^{+}$ cells (Figure 2D). The percentage of Sca $1^{+}$cells in untreated mice was approximately $65 \%-70 \%$. During recovery this percentage decreased to approximately $45 \%$ at days 8 and 12 , but it increased to $65 \%$ by day 16 . In addition to Population I, we observed that a small subset $(-10 \%)$ of Population II cells expressed Sca1. However, this expression was only observed during the recovery stage and corresponded to the expansion of Population II cells. These findings suggest that Population II cells lose their Sca1 expression as differentiation proceeds during recovery.

The kinetics of appearance of the 3 populations suggests a temporal order for their development, where Population I gives rise to Population II, and Population II gives rise to Population III. We tested this model by sorting Population I cells from the spleens of mice transplanted with control bone marrow on day 8 of recovery. The Population I cells were plated in media containing growth factors known to be expressed in the spleen during the recovery period, such as BMP4, SCF, Epo, and Sonic hedgehog (Shh), and hypoxia and cultured cells were analyzed on days 3 and 6 by flow cytometry (Supplemental Figure 5). This analysis shows that Population I cells give rise to Population II cells, which suggests that in vivo the differentiation of Population I is the initial event in the erythroid recovery from bone marrow transplant. We observed very few Population III cells in these cultures, which suggests that their differentiation may require other factors.

Based on flow cytometry and morphology, these 3 populations appear to be distinct cell populations. We next examined the erythroid potential of these cells. In control transplanted mice, we observed the greatest expansion of donor-derived stress BFU-Es on day 8 following transplant (Figure 1). We sorted Populations I, II, and III on day 8 after transplant and assayed their ability to form erythroid colonies in methylcellulose (Figure 2, G-I). Stress BFU-Es are capable of forming BFU-E colonies in cultures supplemented with Epo alone in the absence of other added factors (3). In addition, stress BFU-Es maximally expand when grown in media containing BMP4, SCF, and Epo at $2 \% \mathrm{O}_{2}$. When we examined the colony-forming ability of Population I, we observed that they formed stress BFU-Es. In fact, Population I contained all the stress BFU-Es present in the spleen. Furthermore, these progenitors responded to the different combinations of these factors in a manner that was identical to what we observed previously with stress BFU-Es present in the spleen (5) (Figure 2G). BMP4, SCF, and hypoxia promoted the greatest expansion of stress BFU-Es. These data demonstrate that Population I cells are BMP4-responsive stress BFU-Es. These cells also formed CFU-erythroid (CFU-E) colonies, but unlike bone marrow CFU-Es, which respond primarily to Epo, the production of CFU-Es by Population I cells was greatly enhanced when the cells were grown in media supplemented with BMP4, SCF, and hypoxia (Figure 2H). In contrast to Population I, Population II cells only formed CFU-E colonies. However, these cells exhibited properties more similar to bone marrow CFU-Es, which responded primarily to Epo and hypoxia (Figure 2I). Population III cells failed to form any colonies, which is consistent with their morphology and temporal development as late-stage erythroblasts.

Mice transplanted with $\mathrm{f} / \mathrm{f}$ mutant bone marrow exhibit a defect in the differentiation of stress erythroid progenitors. Unlike control transplanted mice, mice transplanted with $f / f$ mutant bone marrow did not exhibit an expansion of donor-derived stress BFU-Es at day 8 after transplant (Figure 1). We next examined whether the delayed expansion of stress BFU-Es correlated with a defect in the development of Population I stress progenitors in the spleen. Spleen cells were isolated from mice transplanted with $f / f$ mutant bone marrow on days 6, 8, and 12 after transplant (Figure 2, E and F). 
A

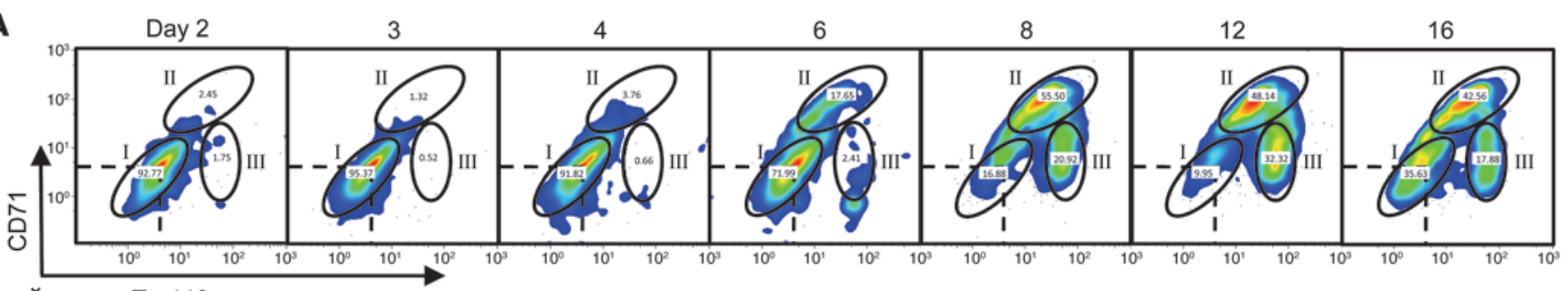
(*) Ter119

B

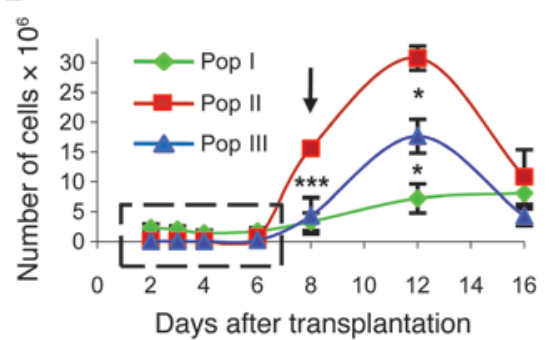

C

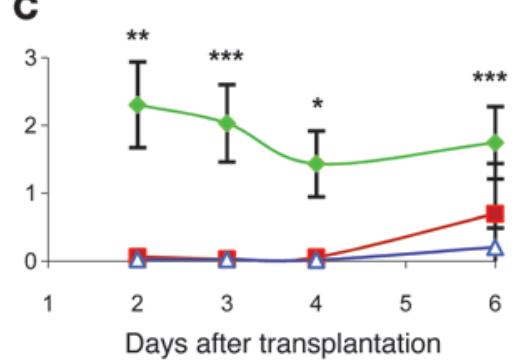

D

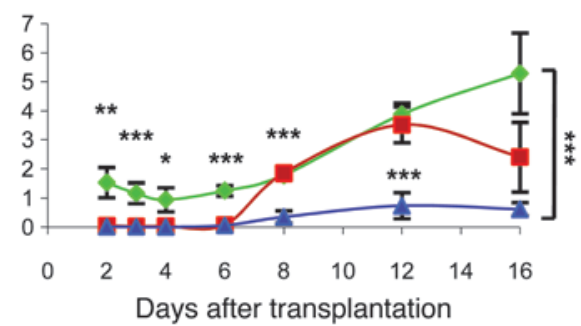

E

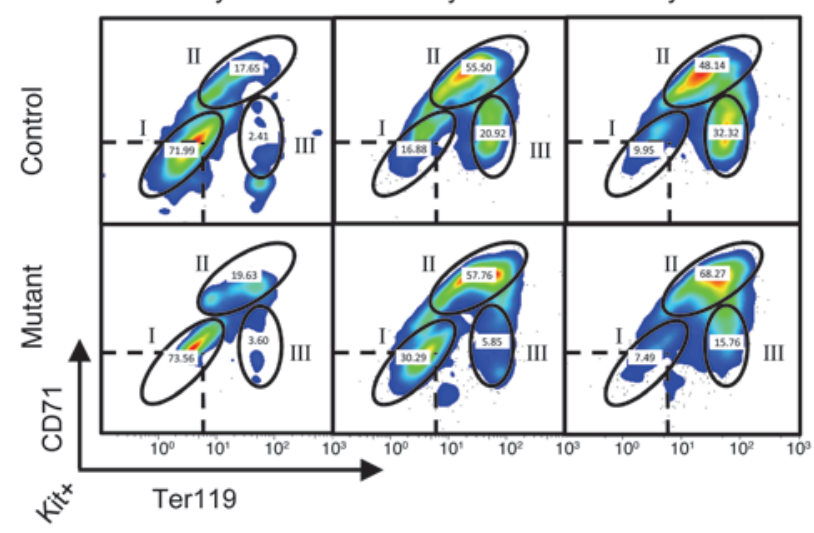

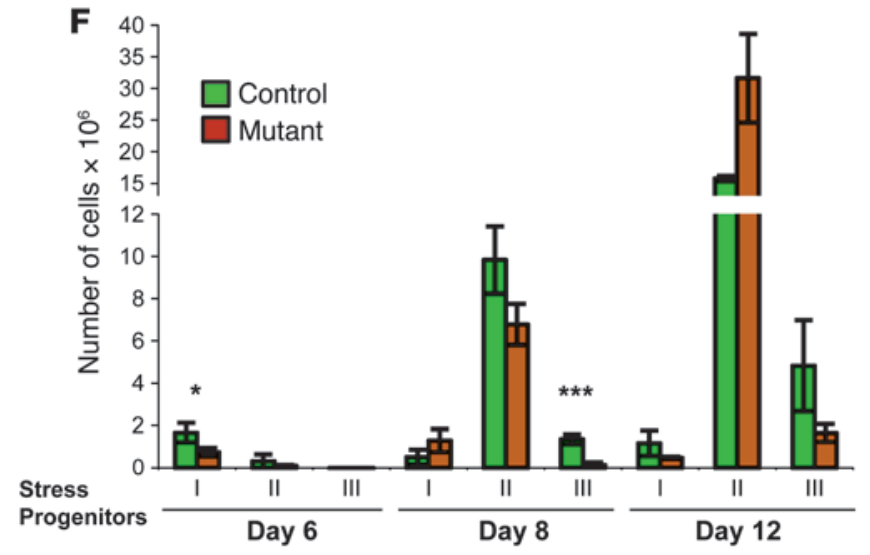

G

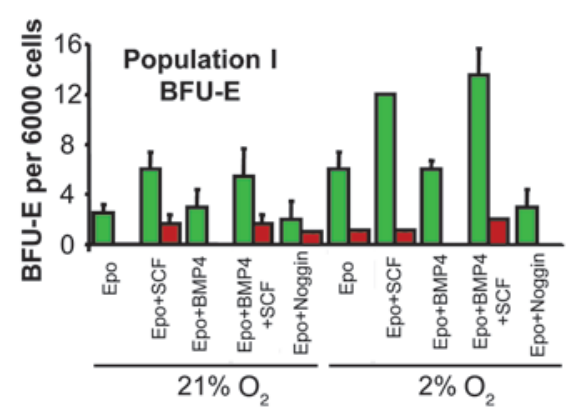

H

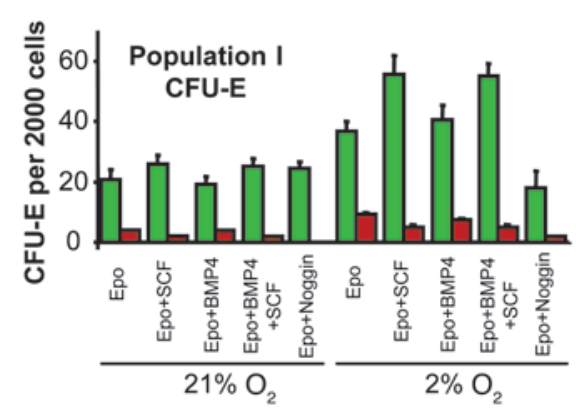

I

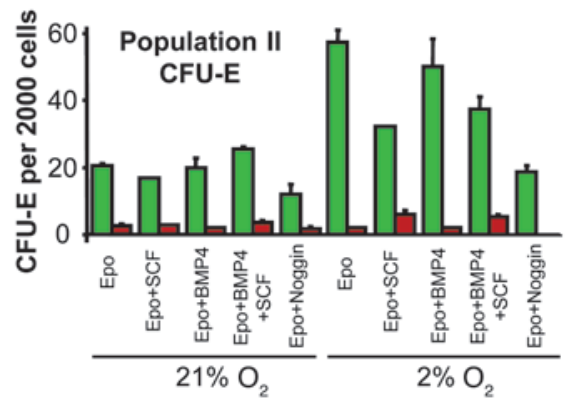

Figure 2

Analysis of stress erythroid progenitor populations by flow cytometry and colony assays. Spleen cells were isolated from CD45.1 mice transplanted with mutant (C57BL/6-f/f) or control (C57BL/6) bone marrow cells. (A) Spleen cells from mice transplanted with control bone marrow cells were labeled with anti-Kit, CD71, and Ter119 antibodies, then gated on Kit+ cells. Expression of CD71 and Ter119 was analyzed by flow cytometry. Populations I-III are indicated. (B) Total number of each stress progenitor population in the spleen after transplant. Data represent the average of 3 mice. (C) Enlarged representation of boxed region in B. (D) Total number of Sca1+ cells in the spleen following transplant. (E) Stress erythroid progenitors in the spleens of mice transplanted with mutant or control bone marrow cells after transplant. Cells were analyzed as described in A. (F) Total number of Populations I-III in mice transplanted with mutant or control bone marrow cells. (G-I) BFU-E colony-forming potential of Population I cells (G) and CFU-E colony-forming potential of Population I (H) and Population II (I) cells isolated by FACS from the spleens of mice transplanted with mutant or control bone marrow cells, on day 8 after transplant. Cells were plated in methylcellulose media containing the indicated growth factors and cultured at $20 \%$ or $2 \% \mathrm{O}_{2}$. For all conditions, control cells produced significantly more BFU-Es or CFU-Es than mutant cells $(P<0.05)$. ${ }^{\star} P<0.05$; ${ }^{\star \star} P<0.01$; ${ }^{\star \star *} P<0.005$. For each time point, at least 3 independent mice were analyzed. 

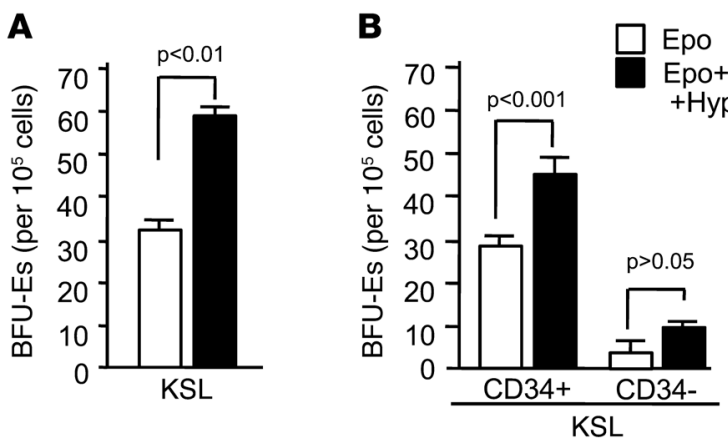

Our analysis shows that early during the recovery phase (day 6) there was a significant reduction in the number of Population I cells in the mice transplanted with $f / f$ bone marrow. However, this defect was not present at day 8 or 12 after transplant. Despite the fact that $f / f$ transplants had similar numbers of Population I cells on day 8 after transplant, they did not exhibit any stress BFU-Es. Indeed, when $f / f$ mutant Population I cells were plated for BFU-Es, few BFU-Es were observed even when cells were plated in media supplemented with BMP4, SCF, and hypoxia (Figure 2G). Similar results were observed when $f / f$ Population I cells were plated for CFU-Es (Figure 2H). Few CFU-E colonies were formed in any of the culture conditions, which demonstrates that Population I cells derived from $f / f$ donors exhibit a delayed development of stress BFU-Es and CFU-Es. Similar to Population I cells, the numbers of Population II progenitor cells at day 8 after transplant were not significantly different than control cells, although at day $12 \mathrm{f} / \mathrm{f}$ transplanted mice appeared to have more cells (Figure 2F). Analysis of CFU-Es from day 8 Population II cells showed that $f / f$ Population II cells were unable to form large numbers of CFU-Es and did not respond to different growth factor conditions like control cells (Figure 2I). Unlike Populations I and II, Population III was significantly reduced at day 8 in the $f / f$ transplanted mice (Figure $2 \mathrm{~F}$ ), which correlates with the delayed production of reticulocytes in the mutant transplants (Supplemental Figure 1).

Bone marrow STR-HSCs $\left(\mathrm{CD} 34^{+} \mathrm{Kit}^{+} \mathrm{Sca} 1^{+} \mathrm{Lin}^{-}\right)$generate stress erythroid progenitors in the spleen. The expression of Kit and Sca1 on Population I progenitors suggested that these cells may be derived from a more primitive progenitor population. We tested whether bone marrow $\mathrm{Kit}^{+} \mathrm{Sca}{ }^{+} \mathrm{Lin}^{-}$(KSL) cells could give rise to donor-derived stress BFU-Es in the spleen on day 8 after transplant. In Figure $3 \mathrm{~A}$, the data show that transplanting $4 \times 10^{5} \mathrm{KSL}$ cells led to the development of stress BFU-Es that responded to BMP4, SCF, and hypoxia. Control experiments using $\mathrm{Kit}^{+} \mathrm{Sca}^{-} \mathrm{Lin}^{-}$donor cells also led to BFU-Es in the spleen, but these progenitors failed to expand in response to BMP4, SCF, and hypoxia. The KSL fraction contained HSCs. This population was further fractionated into longterm reconstituting HSCs (LTR-HSCs), which are CD34-' and STRHSCs, which are $\mathrm{CD}_{3} 4^{+}(23)$. We fractionated bone marrow KSL cells into $\mathrm{CD} 4^{+}$and $\mathrm{CD} 34^{-}$populations and transplanted $2 \times 10^{5}$ donor cells into lethally irradiated mice. On day 8 after transplant, donor-derived cells were sorted from the spleen and plated for stress BFU-Es. The spleens of mice transplanted with CD34+ KSL cells were significantly larger than the spleens of mice transplanted with CD34- KSL cells (Supplemental Figure 6). This difference in spleen size also reflected a difference in the number of stress BFU-Es. $\mathrm{CD} 34^{+} \mathrm{KSL}$ cells generated significant numbers of BFU-Es, which responded to BMP4, SCF, and hypoxia-like stress BFU-Es (Figure 3B).

\section{Figure 3}

Analysis of bone marrow progenitor populations that give rise to BMP4-dependent stress erythroid progenitors in the spleen following transplant. (A) Stress BFU-Es generated by bone marrow KSL cells on day 8 after transplant. (B) Stress BFU-Es produced by bone marrow CD34+ KSLs and CD34- KSLs on day 8 after transplant.

In contrast, the mice transplanted with the CD34- KSL donor cells generated few stress BFU-Es. These data indicate that STR-HSCs generate erythroid stress progenitors during the immediate posttransplant recovery. It is interesting to note that although STRHSCs are CD $34^{+}$, Population I and II cells isolated from the spleen of transplanted mice did not express CD34 (data not shown), which suggests that upon migration to the spleen they lose expression of CD34 but maintain expression of Kit and Sca1.

Stress erythroid progenitors derived from $f / f$ mutant donor bone marrow cells exhibit a delayed expression of Scl and Gata2. Several transcription factors have been shown to play key roles in regulating erythropoiesis. We hypothesized that the defect in BMP4 signaling in the $f / f$ mutant donor cells would impair the expression of factors required for the development or differentiation of stress BFU-Es. We focused on 2 transcription factors, Scl and Gata2, which are known to play a key role in the development of primitive progenitor cells. Scl is required for erythroid short-term radioprotection (24-26). Mice transplanted with $\mathrm{Scl}^{\Delta / \Delta}$ bone marrow fail to generate donor erythrocytes in competitive repopulation assays. Furthermore, CFU-spleen (CFU-S) from these mice do not produce erythroid cells, which phenocopies the defect observed in $f / f C F U-S$ $(26,27)$. These observations suggest that $\mathrm{Scl}$ expression may be a target of BMP4 signaling during the recovery from bone marrow transplant. Gata2, on the other hand, is known to be a target of BMP4 signaling $(28,29)$. Gata2 ${ }^{+/-}$mice exhibit defects in generating progenitor cells in competitive transplants, and the expansion of KSL cells in the bone marrow following 5-FU treatment is significantly impaired (30). Given that $\mathrm{Scl}$ and Gata2 function in concert to regulate erythropoiesis, we analyzed the expression of Scl and Gata2 by quantitative RT-PCR (qRT-PCR) in Population I cells isolated from spleen on day 6 after transplant, which was just prior to the appearance of stress BFU-Es, and on day 8 after transplant, when stress BFU-Es were present. In control transplants, we observed high expression of both Scl and Gata2, but in mice transplanted with $f / f$ mutant bone marrow, Population I cells expressed significantly lower levels of Scl and Gata2 (Figure 4A). This difference in expression was also apparent when we examined Scl protein expression in the spleens of mutant and control transplanted mice. We examined Scl protein expression by staining spleen sections with antibodies to Scl and CD45.2 to identify donor-derived cells. Similar to BMP4, Scl expression was limited to the red pulp of the spleen (Supplemental Figure 7). Scl expression was first observed at day 4, but only a low level of Scl was associated with donor-derived cells. The expression changed dramatically at day 8 when donor-derived cells had expanded in the spleen and nearly all donor-derived cells expressed Scl. By day 12 the expression of Scl was decreased, and expression further decreased by day 20. Simi- 

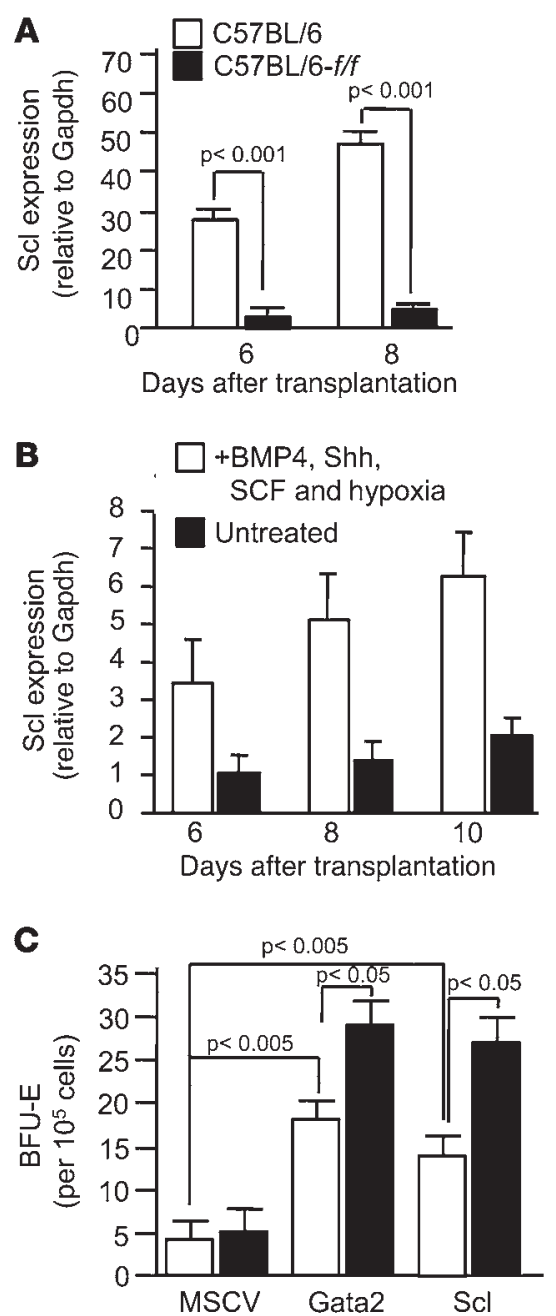
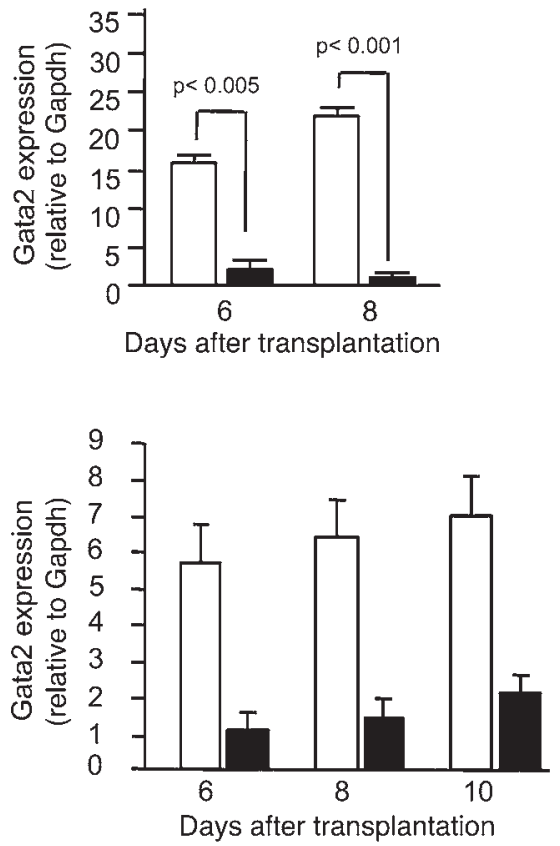

Epo+BMP4

+ hypoxia

Epo

\section{Figure 4}

$\mathrm{Scl}$ and Gata2 expression is regulated by BMP4 during the differentiation of stress erythroid progenitors. (A) qRT-PCR analysis of $\mathrm{Scl}$ (left) and Gata2 (right) expression in Population I progenitor cells sorted from mice transplanted with $\mathrm{f} / \mathrm{f}$ mutant or control bone marrow on days 6 and 8 after transplant. Expression is relative to Gapdh. (B). Population I stress progenitors were sorted on the indicated days after transplant and incubated alone or with BMP4, Shh, SCF, and hypoxia for 2 hours. Scl (left) and Gata2 (right) expression was determined by qRT-PCR. Expression is relative to Gapdh. (C) Rescue of the $f / f$ defect in stress BFU-E development by retroviral expression of $\mathrm{Scl}$ or Gata2. $f / f$ mutant bone marrow cells infected with the indicated viruses were transplanted in mice, and on day 8 after transplant, spleen cells were isolated and plated for stress BFU-Es in methylcellulose media containing either Epo alone or Epo, BMP4, and hypoxia. lar to what we observed with BMP4 expression, donor-derived $f / f$ mutant cells exhibited a delayed expression of SCL. At day 8 very little $\mathrm{Scl}$ expression was present in donor-derived cells (Supplemental Figure 7). At day 12 most donor $f / f$ cells expressed Scl, but at a lower level than control donor cells. However, at day 20 the levels of Scl expression in $f / f$ donor cells were similar to control transplants. Overall, these studies demonstrate that Scl mRNA and protein and Gata2 mRNA are delayed and diminished in mice transplanted with $f / f$ mutant bone marrow, which correlates with the delayed erythroid recovery.

If $\mathrm{Scl}$ and Gata2 are required for the expansion of stress erythroid progenitors, then the signals that promote the expansion of these cells should increase the expression of these factors. We sorted donor-derived cells from mice transplanted with control bone marrow on days 6,8 , and 10 after transplant. The cells were incubated for 2 hours in the presence or absence of all the signals that previously we showed were necessary for the development and expansion of stress BFU-Es - namely, SCF, Bmp4, Shh, and hypoxia (6). The treatment of donor-derived cells with these factors resulted in the upregulation of $S c l$ and Gata2 mRNA expression (Figure 4B). These data show that $\mathrm{Scl}$ and Gata2 are rapidly induced by these signals. In order to test the role of Scl and Gata2 in the expansion of stress BFU-Es in vivo, we infected $f / f$ mutant bone marrow with murine stem cell virus (MSCV) expressing Scl, Gata2, or a MSCV vector control. Twenty-four hours after infection, $\mathrm{GFP}^{+}$cells were sorted and transplanted into irradiated mice. On day 8 after transplant, spleen cells were plated for stress BFU-Es. In Figure 4C, the data show that donor cells infected with either the Scl or the Gata2 virus rescued the defect in the expansion of stress BFU-Es in $f / f$ donor cells. These data demonstrate that $\mathrm{Scl}$ and Gata2 are key targets of BMP4 signaling, which promotes the expansion of stress BFU-Es in the spleen following bone marrow transplant.

Population I cells can provide erythroid short-term radioprotection and self-renew when serially transplanted into irradiated recipients. The coexpression of Kit and Sca1 by Population I and a subset of Population II cells is a surprising finding, given that these cells are erythroid progenitors. Kit and Sca1 expression is associated with stem cells and multipotential progenitor cells $(31,32)$. This observation suggested that these cells may be able to generate multiple cell lineages or potentially self-renew if placed in the correct developmental context. We directly tested this hypothesis by isolating donor-derived Population I and II cells from the spleens of recipient mice on day 8 after transplant and transplanting them into secondary recipients (Figure $5 \mathrm{~A}$ ). Donor $\mathrm{CD} 45.2^{+} \mathrm{Hbb}^{\mathrm{D}}$ bone marrow cells were transplanted into irradiated $\mathrm{CD} 45.1^{+} \mathrm{Hbb}^{\mathrm{S}}$ mice. On day 8 after transplant, donor-derived Population I and II cells were isolated by FACS. Population I or II cells $\left(4 \times 10^{5}\right)$ were transplanted into irradiated $\mathrm{CD} 45.1 \mathrm{Hbb}^{\mathrm{S}}$ recipients, and the recovery followed 
A

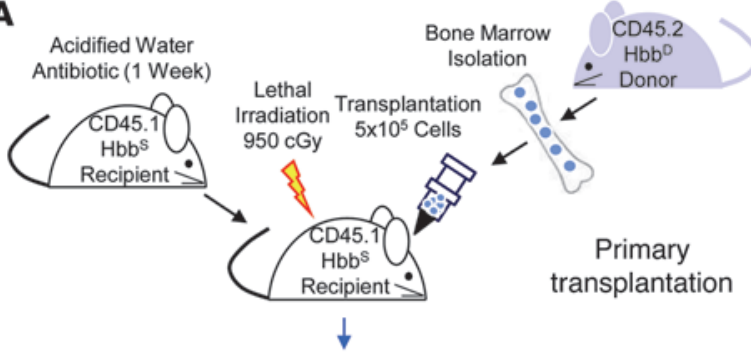

Sort spleen progenitor cells at day 8
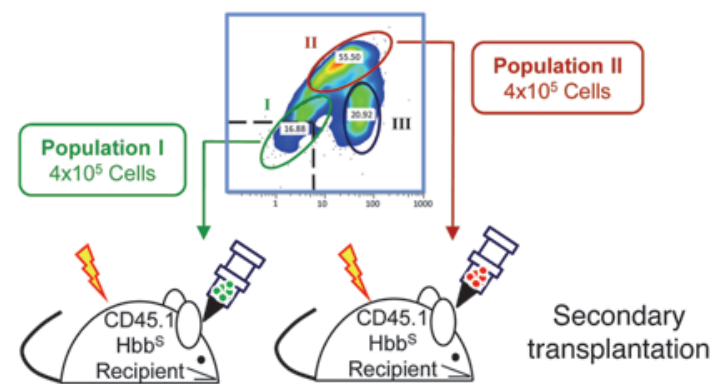

$>42$ Days $\downarrow$

Recovery \& Analysis N No Recovery

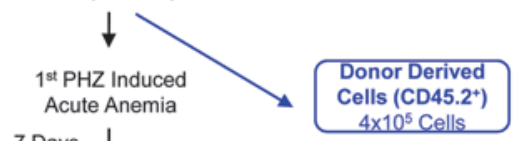

7 Days $\downarrow$

Recovery \& Analysis

56 Days $\downarrow$

$2^{\text {nd }} \mathrm{PHZ}$ Induced

Acute Anemia

7 Days $\downarrow$

Recovery \& Analysis

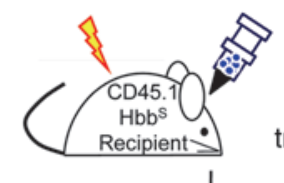

Tertiary transplantation

Recovery \& Analysis
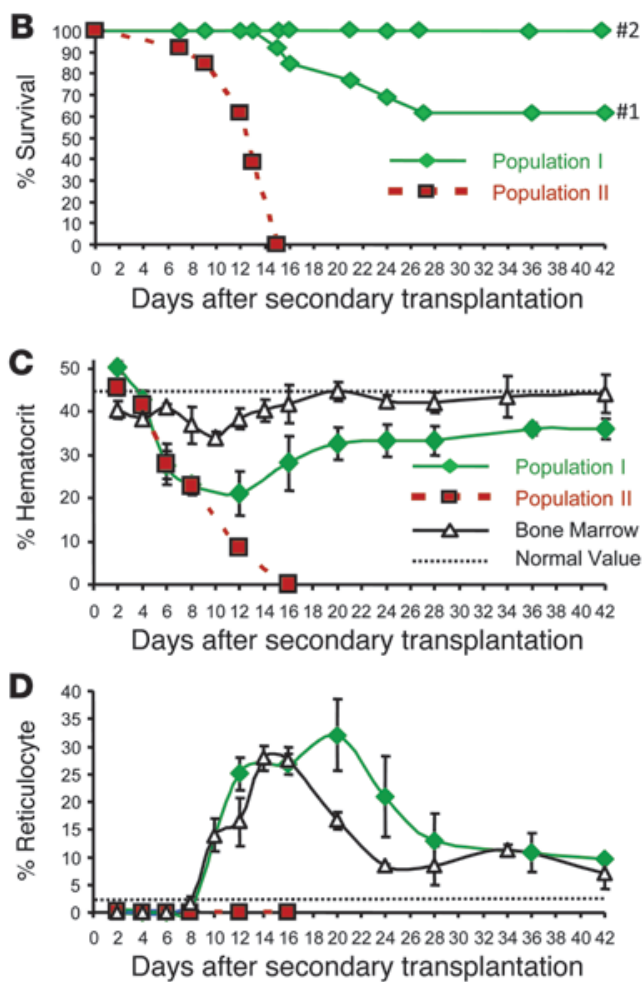

E

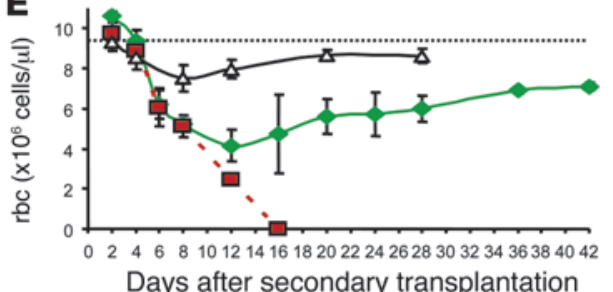

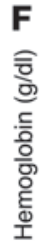

G
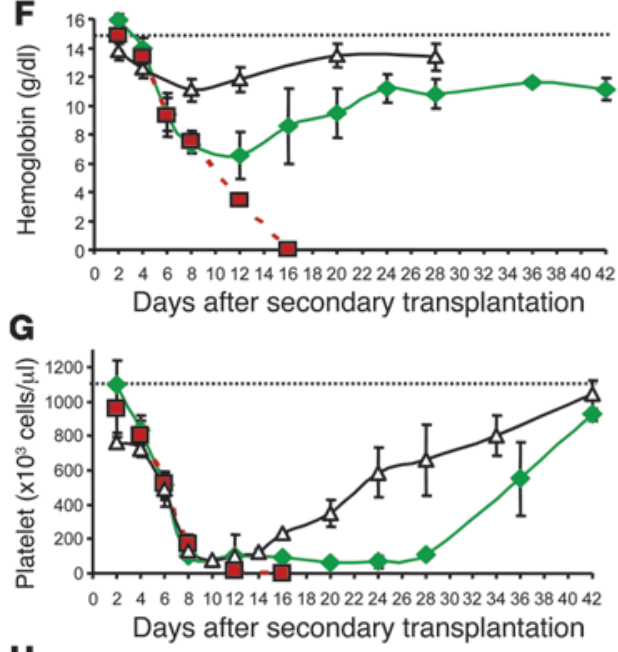

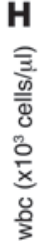

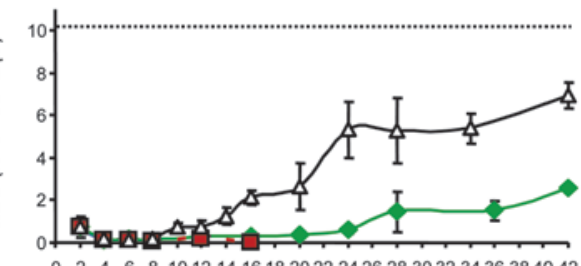

246881012141618202224262830323436384042

Days after secondary transplantation
I

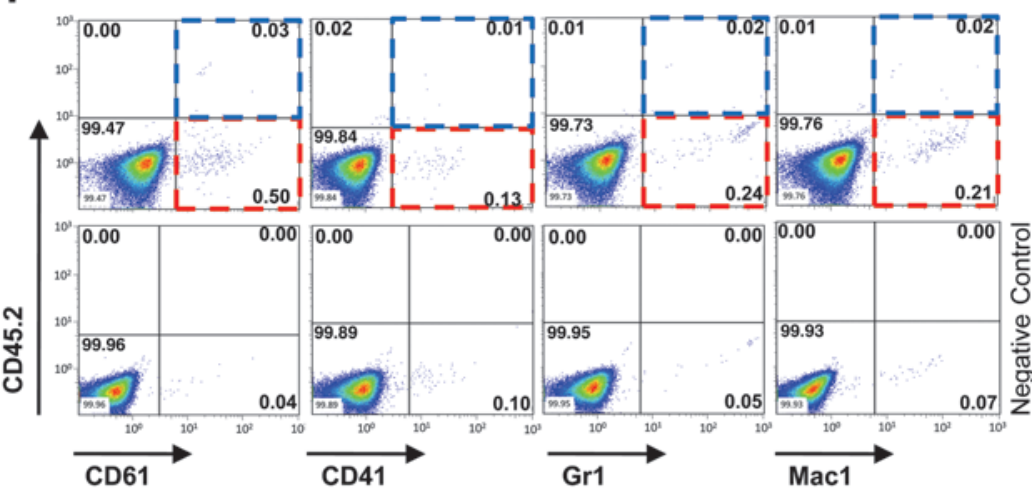

J

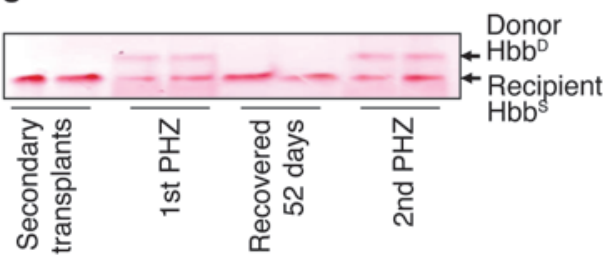

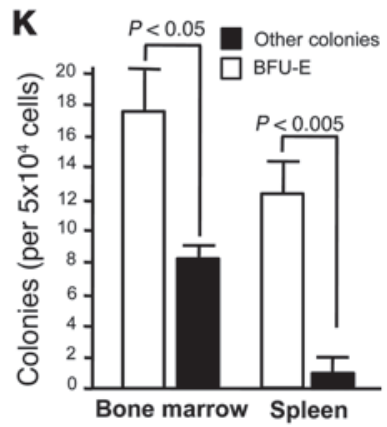




\section{Figure 5}

Population I cells can provide erythroid short-term radio protection when transplanted into secondary recipients. (A) Population I and II cells isolated by FACS from the spleens of CD45.1 mice transplanted with $5 \times 10^{5}$ control $\left(C D 45.2^{+}\right)$bone marrow cells on day 8 after transplant were transplanted into lethally irradiated CD45.1 secondary recipients. The secondary transplants were analyzed for survival $(B)$, hematocrit recovery $(\mathbf{C})$, reticulocyte generation (D), rbc count (E), hemoglobin $(\mathbf{F})$, platelet count $(\mathbf{G})$, and wbc count $(\mathbf{H})$ in Population I and II secondary transplants. The values for the recovery of mice transplanted with unfractionated bone marrow transplants and control untreated mice are indicated. (I) Flow cytometry analysis of peripheral blood mononuclear cells. Cells were harvested 42 days after secondary transplant and stained with anti-CD45.2 and the indicated antibodies (top) or isotype controls (bottom). Data are representative of 3 mice. (J) Analysis of $\mathrm{Hbb}$ alleles on cellulose acetate gels. Secondary transplants ( $>48$ days after transplant) were treated with $\mathrm{PHZ}$ to induce anemia. $\mathrm{Hbb}$ alleles were tested 7 days later ( $1^{\text {st }} \mathrm{PHZ}$ ). The mice were allowed to recover 52 days, then were treated a second time with $\mathrm{PHZ}$, and $\mathrm{Hbb}$ alleles were analyzed 7 days later ( $2^{\text {nd }} \mathrm{PHZ}$ ). (K). Donor-derived cells were sorted from the spleen or bone marrow of secondary recipients. The cells were plated in "complete" methylcellulose media containing SCF+IL-3+IL-6+ Epo. Colonies were counted and scored as BFU-E or "other" myeloid colonies.

over a 6-week period. Mice transplanted with Population II cells exhibited a transient rise in hematocrit (>50\% at day 2) (Figure 5C). However, despite the initial rise in $\mathrm{rbc}$ number, the Population II transplanted mice rapidly developed a lethal anemia and none of the mice survived longer than 15 days (Figure 5B). Population II cells were unable to generate appreciable numbers of reticulocytes, which is consistent with these cells being CFU-Es with little proliferative capacity (Figure 5D). The situation was quite different in the mice transplanted with Population I cells. These mice also exhibited a transient increase in hematocrit ( $>50 \%)$ at day 2 after transplant, which is consistent with our observation that Population I cells exhibit CFU-E activity (Figure 2H). However, unlike the Population II transplanted mice, more than $60 \%$ of the mice transplanted with Population I cells survived in our initial experiments, and by changing the radiation dose from a single dose of 950 rads to 2 doses of 475 rads, we were able to increase the survival of Population I transplanted mice to almost 100\% (Figure 5B). In comparison with mice transplanted with whole bone marrow, the mice transplanted with Population I cells exhibited a more severe anemia, which resolved more slowly (Figure 5C). Using cellulose acetate gel analysis, we observed that from day 16-42, 100\% of the erythrocytes were donor derived, $\mathrm{Hbb}^{\mathrm{D}}$ (data not shown). In Figure $2 \mathrm{~B}$, the data show that the spleens of transplanted mice contain approximately $4 \times 10^{6}$ Population I cells on day 8 after transplant, which is almost 10 -fold more Population I cells than we transplanted in this experiment. Despite the low number of transplanted progenitors, these cells were able to maintain erythropoiesis until surviving recipient stem cells could repopulate the hematopoietic system. Murine erythrocyte half-life is approximately $17-23$ days $(33,34)$. We waited until all the erythrocytes produced by the initial wave of donor-derived stress progenitors would have turned over, and then we tested the expression of $\mathrm{Hbb}$ alleles. At this time, we observed that the erythrocytes in the transplanted mice had reverted to $100 \%$ recipient derived, $\mathrm{Hbb}^{\mathrm{S}}$, which demonstrated that Population I progenitors do not contribute to steady-state erythropoiesis (data not shown).
The most surprising result, however, came when we analyzed the contribution of these cells to other lineages. Platelet recovery was significantly delayed (Figure 5G). We did not observe an increase in platelet number until 28 days after transplant. The recovery of wbc counts was extremely limited, which suggests that these cells have limited myeloid potential (Figure $5 \mathrm{H}$ ). Flow cytometry analysis of peripheral blood leukocytes showed that all the wbc were recipient derived (Figure 5I), including cells expressing the megakaryocyte markers CD41 and CD61. These observations suggest that the few myeloid cells produced are products of recipient-derived progenitors that survived irradiation.

In order to test whether Population I cells could self renew in vivo, we isolated donor-derived spleen cells from secondary transplanted mice and transplanted into tertiary irradiated recipients (Figure 5A). Once again, we observed that the mice survived the transplant and erythroid recovery was similar to that observed in the secondary transplants (Supplemental Figure 8). Hemoglobin analysis showed that erythroid recovery was $100 \%$ donor derived (data not shown). In contrast, analysis of peripheral blood mononuclear cells by flow cytometry indicated that these cells were recipient derived (Supplemental Figure 8). Similar to what we observed in the secondary transplants, surviving recipient stem cells repopulated the mice, and erythrocytes reverted to recipient $\mathrm{Hbb}^{\mathrm{S}}$. These data show that Population I stress erythroid progenitors self renew in vivo and are capable of maintaining erythropoiesis in the absence of HSCs.

Our observation that Population I cells can be serially transplanted shows that they can self renew in vivo. We next tested whether donor-derived Population I cells were maintained in the secondary recipients and could respond to acute anemia even after the mice had been repopulated by surviving HSCs and the erythrocytes had reverted back to recipient-derived $\mathrm{Hbb}^{\mathrm{S}}$. We treated secondary recipients with $\mathrm{PHZ}$ to induced acute anemia, and then 7 days after treatment, we analyzed erythrocytes for Hbb alleles. In Figure 5J, the data show that donor-derived $\mathrm{Hbb}^{\mathrm{D}}$ was induced during the recovery from acute anemia. We extended this observation by allowing the mice to fully recover from the PHZ-induced anemia and the $\mathrm{Hbb}^{\mathrm{D}}$ donor-derived erythrocytes had turned over (52 days). We treated these mice once again with PHZ and analyzed the contribution of donor-derived stress progenitors to the recovery from anemia. Analysis of $\mathrm{Hbb}$ alleles showed that PHZinduced anemia once again led to the production of donor-derived erythrocytes (Figure 5J). These data show that although recipient HSCs have repopulated the mouse and are driving steady-state erythropoiesis, stress erythropoiesis utilized the donor-derived progenitors, which suggests that the stress erythroid response is maintained by a stable stress progenitor cell compartment.

Transplanted Population I progenitor cells preferentially expand in the spleens of recipient mice. Flow cytometry analysis in secondary transplanted mice showed that on day 21 , approximately $97 \%$ of spleen cells were donor derived, while only $4 \%$ were donor derived in the bone marrow (data not shown). These data were similar in tertiary transplants. Our in vivo data show that Population I progenitors were erythroid restricted. We next tested whether donor-derived cells sorted from the spleen or the bone marrow of tertiary transplanted mice exhibited greater developmental potential when plated in methylcellulose media containing a complete set of cytokines (IL-3, IL-6, SCF, and Epo). Donor-derived spleen cells primarily generated only BFU-Es when plated in this media, while donor-derived bone marrow cells generated about 70\% BFU-Es in 


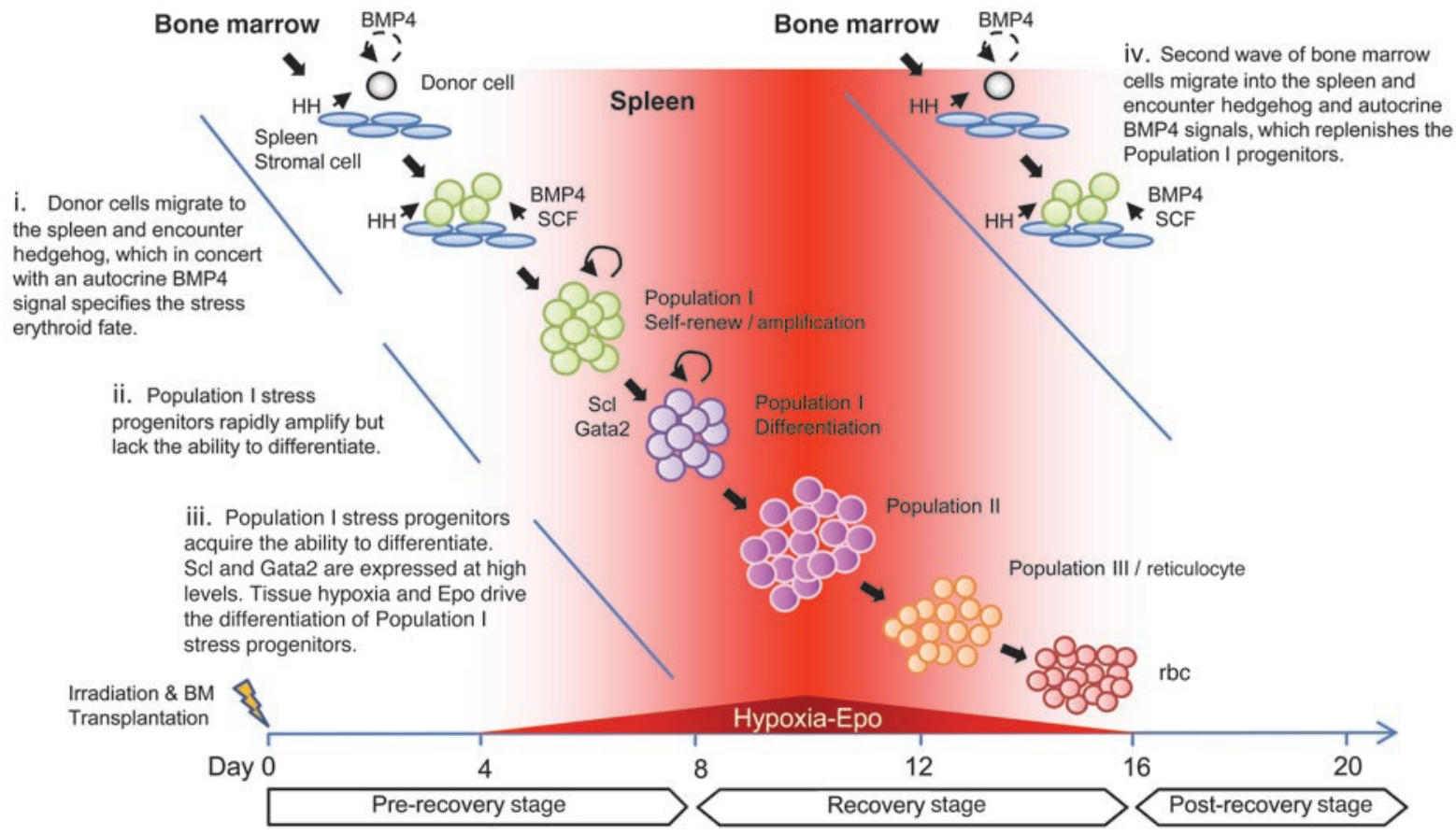

Figure 6

Model for erythroid short-term radioprotection during the recovery from bone marrow transplant. Details are in the text. $\mathrm{HH}$, hedgehog.

addition to other myeloid colonies (Figure 5K). These data show that in vivo and in vitro spleen Population I progenitors are erythroid restricted, but that when these cells are in the bone marrow microenvironment, they can reacquire the potential to form other myeloid lineages.

\section{Discussion}

Our analysis of erythroid short-term radioprotection shows that the BMP4-dependent stress erythropoiesis pathway plays an essential role in the rapid generation of new erythrocytes in the immediate post-transplant period. Our work suggests a new model for stress erythropoiesis (Figure 6). In the initial stage, donor-derived $\mathrm{CD} 34^{+} \mathrm{KSL}$ cells migrate to the spleen. The signals that induce the migration or regulate the homing of progenitor cells to the spleen are not known. In the second stage, these cells adopt the stress erythroid progenitor cell fate. CD $34^{+} \mathrm{KSL}$ cells interact with hedgehog (Dhh and Ihh) in the spleen, which induces them to express BMP4. Hedgehog and BMP4 act in concert to specify the stress erythroid progenitor fate. Once the stress erythroid fate is specified, the stress erythroid progenitors expand in the spleen without differentiating. The results depicted in Figure 2 show that the number of Population I cells in the spleen rapidly increased in the first 8 days after transplant. However, despite the fact that BMP4, SCF, and hedgehog are present in the spleen, we did not observe stress BFU-Es until day 8 after transplant. The development of stress BFU-Es correlates with the nadir in the hematocrit and the induction of Epo expression by the kidney, which is a direct readout of systemic tissue hypoxia (Supplemental Figure 9). This observation is consistent with our previous work showing that stress BFU-Es require BMP4, SCF, and hypoxia to maximally expand in vitro (5). The transition from expanding stress progenitors to stress BFU-Es capable of differentiating marks the last stage in our model. We propose that the switch from proliferation to differentiation requires high-level expression of $\mathrm{Scl}$ and Gata2 and is mediated by tissue hypoxia. Although Epo could play a role in this process, $\mathrm{Epo}^{-/-}$mice still develop BFU-Es, suggesting that Epo is not necessary for BFU-E development (35). The presence of BMP4, SCF, and hypoxia and a rise in serum Epo concentration drives the terminal differentiation of stress BFU-Es and the eventual erythroid recovery of the transplanted mice. Following recovery at day 12 after transplant, we observed a second wave of progenitors migrating into the spleen, as evidenced by the high levels of donor-derived BMP4 expression. These cells replenish the stress erythroid progenitors that have recently differentiated (6). However, the lack of a hypoxia signal prevents their differentiation so they remain dormant.

Our analysis points to a defect in the differentiation of stress progenitors as the cause of the delayed erythroid recovery in the mice transplanted with $f / f$ mutant bone marrow. The $f$ mutation causes a defect in splicing that results in the production of a dominant-negative form, which can inhibit signaling by $\operatorname{Smad} 1,-5$, and $-8(3,12)$. On day 8 after transplant, the numbers of Population I progenitors were equivalent in the mutant and control transplants, but the mutant transplants lacked stress BFU-Es. We hypothesize that the progression from expanding stress erythroid progenitors to stress BFU-Es capable of terminal differentiation relies on the expression of Scl and Gata2. Analysis of the expression of these genes in Population I cells showed that mutant progenitor cells exhibited significantly lower Scl and Gata2 expression. Treatment of control donor-derived cells isolated from spleen with BMP4, hedgehog, $\mathrm{SCF}$, and hypoxia induced the expression of Scl and Gata2 in 2 hours, suggesting that these genes are direct or indirect targets of these signals. The importance of these genes in the development of stress BFU-Es is underscored by the data in Figure 4, which show that retroviral expression of $\mathrm{Scl}$ or Gata2 rescues the delay in stress 
BFU-E development. These data are consistent with previous work showing that $\mathrm{Scl}$ expression can be regulated by hedgehog signaling (36) and that Gata2 is a BMP4-inducible gene $(28,29)$. Further analysis will be needed to establish the relationship between $\mathrm{Scl}$ and Gata2 expression and BMP4 and hedgehog signaling in the spleen during the recovery from bone marrow transplant.

Surprisingly, mice transplanted with $f / f$ mutant bone marrow also exhibited a defect in BMP4 expression by the spleen stroma (Supplemental Figure 3). Our recent work showed that BMP4 is regulated by hypoxia in the spleen, and we have identified 2 hypoxia-responsive elements in the BMP4 gene (37). However, hypoxia is not the whole story. Mock transplanted mice exhibit only a transient increase in BMP4 expression despite the low hematocrits (data not shown). This observation suggests that the regulation of BMP4 expression is more complex, and we propose that the donor cells make a signal that cooperates with hypoxia to maintain the expression of BMP4.

The most surprising result of our study is the observation that purified Population I cells can maintain erythropoiesis when transplanted into secondary and tertiary recipients, without contribution to other lineages, until surviving recipient-derived HSCs expand and repopulate the mice. These serial transplant experiments clearly demonstrate that Population I cells self renew. Furthermore, donor stress progenitor cells are maintained in the secondary and tertiary transplants even after the recipient HSCs repopulate the mice. The donor stress progenitor cells primarily populate the spleen, but a small percentage of donor-derived cells are present in the bone marrow. Donor cells in the spleen are restricted to the erythroid lineage, consistently generating only BFU-Es in colony assays. Although donor cells in the bone marrow preferentially develop into BFU-Es, they can also generate other lineages. This observation suggests that spleen microenvironment restricts the potential of the cells. The donor-derived stress progenitors are functional in secondary and tertiary recipients and are capable of generating new erythrocytes in response to PHZ-induced acute anemia despite the fact that the surviving recipient HSCs have repopulated the mice. This donor-derived stress response is maintained through multiple anemic challenges, which further underscores their self-renewal capability. These observations suggest that Population I stress erythroid progenitors, and potentially STR-HSCs, may represent a stress response compartment that is maintained separately from LTR-HSCs.

The directed differentiation of HSCs or multipotential progenitor cells (MPPs) at times of physiological stress was previously observed when treatment of HSCs and MPPs with TLR ligands resulted in the development of macrophages, granulocytes, and dendritic cells by a process that bypassed the normal differentiation pathways (38). Nagai et al. suggested that the response of early progenitors to these signals during infection may promote the rapid replenishment of innate immune cells (38). In our case, anemic stress alters the spleen microenvironment, which induces the differentiation of STR-HSCs. These STR-HSCs rapidly develop into an erythroid-lineage restricted stress progenitor, apparently without proceeding through the intervening stages of development. These data suggest that at times of acute life-threatening conditions, the hematopoietic system can adapt to rapidly produce new mature cells to alleviate the stress condition.

Our analysis presented here focused on murine stress erythropoiesis, which leaves the question of whether this pathway functions in humans. The analysis of human stress erythropoiesis has been limited to studies of erythropoiesis in severely anemic patients, which can be complicated by indirect effects of disease pathology. Despite these difficulties, some general aspects of human stress erythropoiesis have been reported. Similar to murine stress erythropoiesis, human stress erythropoiesis exhibits properties of fetal erythropoiesis $(39,40)$. The expression of fetal antigens and fetal hemoglobin ( $\mathrm{HbF}$ ) has been observed following bone marrow transplant (41-43) and in patients with anemia (44-46). In addition, treatment of non-human primates with $\mathrm{PHZ}$ to induce anemia results in increased expression of $\mathrm{HbF}$ (47-49). Analysis of peripheral blood and bone marrow progenitors in patients with sickle cell anemia identified a putative stress erythroid progenitor that expressed CD34, Kit, CD71, and glycophorin A on their surface. These cells readily produced $\mathrm{HbF}^{+}$erythrocytes in culture, and the production $\mathrm{F}$ cells is increased when the cells are incubated at low $\mathrm{O}_{2}$ (50). It is tempting to speculate that these cells are the human equivalent to the $\mathrm{Kit}^{+} \mathrm{Sca} 1^{+} \mathrm{CD} 71^{\mathrm{lo}} \mathrm{Ter} 119^{\text {lo }}$ Population I stress erythroid progenitors we observe in mice. More work will be necessary to determine the similarities between these 2 systems.

\section{Methods}

Mice. C57BL/6-f, C57BL/6 (CD45.2), and congenic B6.SJL-Ptprc ${ }^{a}$ Pep $3^{b} /$ BoyJ (CD45.1) mice were purchased from The Jackson Laboratory. All mice were approximately $6-10$ weeks old; controls were age matched. All procedures were approved by the IACUC of the Pennsylvania State University.

Complete blood analysis. Peripheral blood was obtained by retro-orbital sinus through heparin-coated microhematocrit tubes and transferred to tubes coated with EDTA for complete blood cell (CBC) analysis. CBC analysis was determined using an Advia 120 multi-species whole-blood analyzer (Bayer).

Flow cytometry and cell sorting. Single-cell suspensions of splenocytes, bone marrow, and peripheral blood were isolated and labeled (5) with different combinations of antibodies: anti-Fc $\gamma$ RII/III, CD71-FITC, Ter119-PE, CD45.1-FITC, CD45.2-FITC, CD61-PE, and CD31-FITC (BD Biosciences Pharmingen), anti-Kit-Alexa Fluor 647, and Sca1-Pacific Blue (BioLegend), anti-Kit-PE-Alexa Fluor 610, and Sca1-Alexa Fluor 647 (Invitrogen). After labeling, cells were washed with $2 \%$ fetal calf serum in PBS. Flow cytometry analysis was done using a FC500 Benchtop Flow Cytometer (BeckmanCoulter) with CXP software. The percentage of dead cells was measured by propidium iodide staining. All the data were analyzed with FlowJo software (Tree Star). Cell populations were sorted as Kit ${ }^{+} \mathrm{CD} 71^{-/ \mathrm{lo}} \mathrm{Ter} 119^{-/ \mathrm{lo}}$ (Population I), $\mathrm{Kit}^{+} \mathrm{CD} 71^{\text {hi Ter } 119} 9^{\text {med }}$ (Population II), and $\mathrm{Kit}^{+} \mathrm{CD} 71^{-/ \text {med }}$ Ter119 ${ }^{\text {hi }}$ (Population III) by using a Cytopeia inFlux V-GS Cytometry Workbench with Spigot software. Some cell populations were sorted and spun onto slides using a Cytospin 3 centrifuge (Shandon) at $33 \mathrm{~g}$ for 10 minutes, fixed in methanol for 10 minutes, and subjected to $\mathrm{H} \& \mathrm{E}$, neutral benzidine, or Giemsa staining according to the manufacturer's recommendations.

Transplantation assays. For primary transplantation, $1 \times 10^{5}$ and $5 \times 10^{5}$ bone marrow mononuclear cells from C57BL/6 (CD45.2+) and C57BL/6-f/f $\left(\mathrm{CD} 45.2^{+}\right)$mice were isolated and transplanted into the retro-orbital sinus of lethally irradiated (9.5-Gy) B6.SJL-Ptprc ${ }^{a} P e p 3^{b} /$ BoyJ (CD45.1 $1^{+}$) recipients. Prior to transplantation all recipients were treated with acidified water ( $\mathrm{pH}$ 2.5-3.0) and antibiotics for 1 week. Reconstitution was monitored by FACS analysis of bone marrow and spleen cells at post-transplantation time points. The primary transplantation experiments were repeated several times under the same conditions for different analysis purposes. For secondary transplantation experiments, Population I and II (CD45.2 $)$ cells were sorted from spleens of primary transplanted animals (CD45. $\left.1^{+}\right)$at day 8 and $4 \times 10^{5}$ sorted cells were transplanted into lethally irradiated CD $45.1^{+}$ secondary recipients. A cohort of secondary recipients was used for further analysis. The rate of reconstitution was measured by FACS analysis. 
Colony assays. Donor-derived splenocytes were isolated FACS on the indicated days from B6.SJL-Ptprc ${ }^{a}$ Pep3 $3^{b} /$ BoyJ $\left(C D 45.1^{+}\right)$mice transplanted with C57BL/ 6 or C57BL/6-f/f bone marrow. Nucleated splenocytes $\left(2 \times 10^{6} / \mathrm{ml}\right)$ were plated in methylcellulose media (StemCell Technologies) containing $3 \mathrm{U} / \mathrm{ml}$ Epo (US Biological). Stress BFU-Es were scored as described after 5 days incubation (5). For the analysis of the potential of Populations I, II, and III to form BFU-Es and CFU-Es, spleen cells were isolated from B6.SJL-Ptprc ${ }^{a}$ $\mathrm{Pep}^{6}$ /BoyJ $\left(\mathrm{CD} 45.1^{+}\right)$mice transplanted with C57BL/6 or C57BL/6- $f / f$ bone marrow by FACS on day 8 after transplant. The indicated number of cells was plated in methylcellulose media (StemCell Technologies) containing $3 \mathrm{U} / \mathrm{ml}$ Epo (US Biological) with or without $50 \mathrm{ng} / \mathrm{ml} \mathrm{SCF}, 15 \mathrm{ng} / \mathrm{ml} \mathrm{BMP} 4$, and/ or Noggin (R\&D Systems), as indicated in the figures. Stress BFU-Es were scored as described after 5 days of incubation (5), and CFU-Es were scored after 2 days. For assays done at $2 \% \mathrm{O}_{2}$, plates were incubated in a modular incubator chamber (Billups-Rothenberg) as previously described (5).

RT-PCR and gene expression analysis. RNA was isolated from the cells or tissues indicated in the figures using TRIzol reagent (Invitrogen) according to the manufacturer's instructions. cDNA was prepared using the SuperScript-II system for RT-PCR (Invitrogen). qRT-PCR was done using Taqman assays for Scl (Mm00441665-A1), Gata2 (Mm00492302-g1), and Gapdh (Mm03302249-g1) from Applied Biosystems according to the manufacturer's instructions. The qRT-PCR analysis was done using an ABI 7300 Real-Time PCR system.

Statistics. For all experiments with error bars, standard deviation was calculated to indicate the variation within each experiment. Mean differences between groups were examined by Student's $t$ test (2-tailed). Statistical significance was taken at values of $P<0.05$.

\section{Acknowledgments}

We thank Susan Magargee, Elaine Kunze, and Nicole Zembower in the flow cytometry facility for their excellent help and advice on cell sorting, Jeff Dodds for his help with histology and in developing the bone marrow transplant protocol, and Ruth Haldeman in the electron microscopy facility. Gerd Blobel provided the MSCV-Scl and MSCV-Gata2 virus. We also thank the members of the Paulson laboratory for their support. Special thanks to Gholamreza Sharifi. This work was funded by a grant from the National Blood Foundation (to R.F. Paulson), seed funding (to R.F. Paulson), NIH grant DK080040 (to R.F. Paulson), and graduate student competitive research awards (to O.F. Harandi and D.-C. Wu) from the College of Agricultural Sciences at Pennsylvania State University.

Received for publication September 29, 2009, and accepted in revised form September 15, 2010.

Address correspondence to: Robert F. Paulson, Center for Molecular Immunology and Infectious Disease, Department of Veterinary and Biomedical Sciences, 115 Henning Building, Pennsylvania State University, University Park, Pennsylvania 16802, USA. Phone: 814.863.6306; Fax: 814.863.6140; E-mail: rfp5@psu.edu.

Omid F. Harandi's present address is: Program in Molecular Medicine, University of Massachusetts Medical School, Worcester, Massachusetts, USA.
1. Longmore GD. A unique role for Stat5 in recovery from acute anemia. JClin Invest. 2006;116(3):626-628.

2. Socolovsky M. Molecular insights into stress erythropoiesis. Curr Opin Hematol. 2007;14(3):215-224.

3. Lenox L, Perry J, Paulson R. BMP4 and Madh5 regulate the erythroid response to acute anemia. Blood. 2005;105(7):2741-2748.

4. Lenox LE, Shi L, Hegde S, Paulson RF. Extramedullary erythropoiesis in the adult liver requires BMP-4/Smad5-dependent signaling. Exp Hematol. 2009;37(5):549-558

5. Perry J, Harandi O, Paulson R. BMP4, SCF and Hypoxia cooperatively regulate the expansion of murine stress erythroid progenitors. Blood. 2007;109(10):4494-4502.

6. Perry JM, Harandi OF, Porayette P, Hegde S, Kannan AK, Paulson RF. Maintenance of the BMP4 dependent stress erythropoiesis pathway in the murine spleen requires hedgehog signaling. Blood. 2009;113(4):911-918.

7. Porayette P, Paulson RF. BMP4/Smad5 dependent stress erythropoiesis is required for the expansion of erythroid progenitors during fetal development. Dev Biol. 2008;317(1):24-35

8. Coleman D, Russell E, Levin E. Enzymatic studies of the hemopoietic defect in flexed mice. Genetics. 1969;61(3):631-642.

9. Gruneberg H. The anaemia of the flexed-tail mouse (Mus musculus L.) I. Static and dynamic haematology. J Genet. 1942;43:45-68.

10. Mixter R, Hunt H. Anemia in the flexed tailed mouse, Mus musculus. Genetics. 1933;18(4):367-387.

11. Russell E. Hereditary anemias of the mouse: a review for geneticists. Adv Genet. 1979;20:357-459.

12. Hegde S, et al. An intronic sequence mutated in flexed-tail mice regulates splicing of Smad5. Mamm Genome. 2007;18(12):852-860

13. Perry JM, Harandi OF, Porayette P, Hegde S, Kannan AK, Paulson RF. Maintenance of the BMP4 dependent stress erythropoiesis pathway in the murine spleen requires hedgehog signaling. Blood. 2009; 113(4):911-918.

14. Jones RJ, et al. Characterization of mouse lympho- hematopoietic stem cells lacking spleen colonyforming activity. Blood. 1996;88(2):487-491.

15. Jones RJ, Wagner JE, Celano P, Zicha MS, Sharkis SJ. Separation of pluripotent haematopoietic stem cells from spleen colony-forming cells. Nature. 1990;347(6289):188-189.

16. Singbrant $S$, et al. Canonical BMP signaling is dispensable for hematopoietic stem cell function in both adult and fetal liver hematopoiesis, but essential to preserve colon architecture. Blood. 2010 115(23):4689-4698.

17. Singbrant $S$, et al. Smad5 is dispensable for adult murine hematopoiesis. Blood. 2006; 108(12):3707-3712.

18. Baumann CI, Bailey AS, Li W, Ferkowicz MJ, Yoder MC, Fleming WH. PECAM-1 is expressed on hematopoietic stem cells throughout ontogeny and identifies a population of erythroid progenitors. Blood. 2004;104(4):1010-1016.

19. Na Nakorn T, Traver D, Weissman IL, Akashi K. Myeloerythroid-restricted progenitors are sufficient to confer radioprotection and provide the majority of day 8 CFU-S. J Clin Invest. 2002;109(12):1579-1585.

20. Subramanian A, Hegde S, Porayette P, Yon M, Hankey P, Paulson RF. Friend virus utilizes the BMP4 dependent stress erythropoiesis pathway to induce erythroleukemia. J Virol. 2008;82(1):382-393.

21. Wojchowski DM, et al. Erythropoietin-dependent erythropoiesis: New insights and questions. Blood Cells Mol Dis. 2006;36(2):232-238.

22. Zhang J, Socolovsky M, Gross AW, Lodish HF. Role of Ras signaling in erythroid differentiation of mouse fetal liver cells: functional analysis by a flow cytometry-based novel culture system. Blood. 2003;102(12):3938-3946.

23. Osawa M, Hanada K, Hamada H, Nakauchi H. Long-term lymphohematopoietic reconstitution by a single CD34-low/negative hematopoietic stem cell. Science. 1996;273(5272):242-245.

24. Curtis DJ, Hall MA, Van Stekelenburg LJ, Robb L, Jane SM, Begley CG. SCL is required for normal function of short-term repopulating hematopoietic stem cells. Blood. 2004;103(9):3342-3348.
25. Hall MA, et al. The critical regulator of embryonic hematopoiesis, SCL, is vital in the adult for megakaryopoiesis, erythropoiesis, and lineage choice in CFU-S12. Proc Natl Acad Sci U S A. 2003; 100(3):992-997.

26. Hall MA, et al. Functional but abnormal adult erythropoiesis in the absence of the stem cell leukemia gene. Mol Cell Biol. 2005;25(15):6355-6362.

27. Cole R, Regan T. Haematopoietic Progenitor cells in the prenatal conegenitally anaemic "Flexed-tail" (f/f) mice. Br J Haematol. 1976;33(3):387-394.

28. Lohmann F, Bieker JJ. Activation of Eklf expression during hematopoiesis by Gata2 and Smad5 prior to erythroid commitment. Development. 2008;135(12):2071-2082.

29. Lugus JJ, et al. GATA2 functions at multiple steps in hemangioblast development and differentiation. Development. 2007;134(2):393-405.

30. Ling KW, et al. GATA-2 plays two functionally distinct roles during the ontogeny of hematopoietic stem cells. J Exp Med. 2004;200(7):871-882.

31. Holmes C, Stanford WL. Concise review: stem cell antigen-1: expression, function, and enigma. Stem Cells. 2007;25(6):1339-1347.

32. Spangrude GJ, Heimfeld S, Weissman IL. Purification and characterization of mouse hematopoietic stem cells. Science. 1988;241(4861):58-62.

33. Goodman JW, Smith LH. Erythrocyte life span in normal mice and in radiation bone marrow chimeras. Am J Physiol. 1961;200:764-770.

34. Hoffmann-Fezer G, et al. Biotin labeling as an alternative nonradioactive approach to determination of red cell survival. Ann Hematol. 1993;67(2):81-87.

35. Wu H, Liu X, Jaenisch R, Lodish HF. Generation of committed erythroid BFU-E and CFU-E progenitors does not require erythropoietin or the erythropoietin receptor. Cell. 1995;83(1):59-67.

36. Hochman E, Kinston S, Harmelin A, Gottgens B, Izraeli S. The SCL 3' enhancer responds to Hedgehog signaling during hemangioblast specification. Exp Hematol. 2006;34(12):1643-1650.

37. Wu DC, Paulson RF. Hypoxia regulates BMP4 expression in the murine spleen during the recovery 
from acute anemia. PLoS One. 2010;5(6):e11303.

38. Nagai Y, et al. Toll-like receptors on hematopoietic progenitor cells stimulate innate immune system replenishment. Immunity. 2006;24(6):801-812.

39. Alter BP. Fetal erythropoiesis in stress hematopoiesis. Exp Hematol. 1979;7 suppl 5:200-209.

40. Stamatoyannopoulos G, Veith R, Galanello R, Papayannopoulou T. Hb F production in stressed erythropoiesis: observations and kinetic models. Ann N Y Acad Sci. 1985;445:188-197.

41. Galanello R, et al. Erythropoiesis following bone marrow transplantation from donors heterozygous for beta-thalassaemia. Br J Haematol. 1989; 72(4):561-566.

42. Meletis J, et al. 'Fetal' erythropoiesis following bone marrow transplantation as estimated by the number of F cells in the peripheral blood. Bone Marrow
Transplant. 1994;14(5):737-740.

43. Weinberg RS, Schofield JM, Lenes AL, Brochstein J, Alter BP. Adult 'fetal-like' erythropoiesis characterizes recovery from bone marrow transplantation. BrJ Haematol. 1986;63(3):415-424.

44. Bank A. Regulation of human fetal hemoglobin: new players, new complexities. Blood. 2006; 107(2):435-443.

45. Link MP, Alter BP. Fetal-like erythropoiesis during recovery from transient erythroblastopenia of childhood (TEC). Pediatr Res. 1981;15(7):1036-1039.

46. Papayannopoulou T, Vichinsky E, Stamatoyannopoulos G. Fetal $\mathrm{Hb}$ production during acute erythroid expansion. I. Observations in patients with transient erythroblastopenia and post-phlebotomy. Br J Haematol. 1980;44(4):535-546.

47. DeSimone J, Biel M, Heller P. Maintenance of fetal hemoglobin (HbF) elevations in the baboon by prolonged erythropoietic stress. Blood. 1982; 60(2):519-523.

48. DeSimone J, Biel SI, Heller P. Stimulation of fetal hemoglobin synthesis in baboons by hemolysis and hypoxia. Proc Natl Acad Sci U S A. 1978; 75(6):2937-2940

49. DeSimone J, Heller P, Adams JG. Hemopoietic stress and fetal hemoglobin synthesis: comparative studies in vivo and in vitro. Blood. 1979; 54(5):1176-1181.

50. Luck L, Zeng L, Hiti AL, Weinberg KI, Malik P. Human CD34(+) and CD34(+)CD38(-) hematopoietic progenitors in sickle cell disease differ phenotypically and functionally from normal and suggest distinct subpopulations that generate F cells. Exp Hematol. 2004;32(5):483-493. 